\title{
Vitamin A Compound
}

National Cancer Institute

\section{Source}

National Cancer Institute. Vitamin A Compound. NCI Thesaurus. Code C938.

A compound containing vitamin A, a natural, fat-soluble vitamin that plays a vital role in a variety of physiologic processes including immune function, vision, reproduction, and cellular growth and differentiation. Upon administration, the various forms of vitamin A are solubilized into micelles in the intestinal lumen and absorbed by duodenal mucosal cells. 